\title{
Finger Pain out of Proportion to Exam: A Case of Occult Hydrofluoric Acid Toxicity
}

\section{Robert Anthony Bassett ${ }^{*}$}

Division of Medical Toxicology, Department of Emergency Medicine, Einstein Medical Center, Philadelphia, pa 19147, USA

\begin{abstract}
Background: Hydrofluoric acid (HFA) toxicity can have significant morbidity and even mortality associated with seemingly small exposures. As a result of a delayed time to symptom onset, low concentration exposures may present as a diagnostic dilemma.
\end{abstract}

Objective: To demonstrate an atypical case presentation of HFA exposure, the inherent diagnostic difficulty, and subtle clues to confirming the diagnosis.

Case Report: We present the case of a 21 year old male day laborer who presented to the emergency department 12 hours after exposure to a cleaning agent used to "polish" metal with significant finger pain, minimal physical exam findings, and refractory to analgesics. He demonstrated hypocalcemia and hypomagnesemia. The patient reported symptom resolution after treatment with topical calcium gluconate.

Conclusion: This case demonstrates the importance of considering HFA toxicity in any patient who presents with pain, minimal physical exam findings, after exposure to an unknown chemical cleaning agent.Screening for the common electrolyte depletion associated with HFA toxicity may help to confirm the diagnosis as well as guide therapy.

Keywords: Hydrofluoric acid; Occult toxicity; Chemical burns; Topical calcium gluconate; Hand trauma

\section{Introduction}

Hydrofluoric acid toxicity can have significant morbidity and even mortality associated with seemingly small exposures.The impact of significant electrolyte disturbances can lead to sudden cardiac collapse. Owing to a delayed time to symptom onset, low concentration exposures may present as a diagnostic dilemma.The patient's lack of association between exposure and symptoms may eliminate key historical information. Additionally the lack of physical exams findings associated with low concentration HFA exposure adds to the challenges of making the correct diagnosis.We present a case of a 21 -year-old male who presented to the emergency department with finger pain out of proportion to physical exam findings and no clear exposure to HFA. This case illustrates the importance of considering HFA toxicity in all unknown chemical exposures.

\section{Case Report}

A 21-year-old male presented to the emergency department 12 hours after using a cleaning agent that was supplied by his employer to shine metal trim. The patient reported using non-waterproof gloves and no direct contact with the cleaning agent.Approximately one hour after using the cleaning agent, the patient reported feeling a painful prickly sensation that progressively worsened.The patient reported "multiple" visits to the sink to perform standard hand washing.The patient attempted using 1 dose of an oral non-steroidal anti inflammatory drug and a topical antibiotic ointment to sooth his discomfort without relief. Over the course of the next 8 hours the pain became intolerable and the patient presented to the hospital.

Upon arriving at the emergency department the patient's vital signs included a blood pressure $143 / 102$, heart rate 104 , respiratory rate 22 per minute, temperature of $36.5^{\circ} \mathrm{C}$, and an oxygen saturation of $99 \%$ on room air.His physical exam was unremarkable except for his hands. The palmar surface of all 5 distal digits on the right hand and the palmar surface of the distal left thumb demonstrated mild erythema.The first 3 digits on the right also demonstrated some minimal whitening on the distal tips.There was no edema, blisters or ulcerations.Neurologically the patient was completely intact including 2-point discrimination over the affected area.

The patient empirically received an appropriate weight-based dose of morphine and ondansetron and a 1-literintravenous bolus of $0.9 \%$ $\mathrm{NaCl}$ solution. Basic laboratory tests were performed that included a complete blood count, a comprehensive metabolic profile, and coagulation studies.Initial studies revealed a white blood cell count of $14.32 \times 10^{9} / \mathrm{L}$, sodium $139 \mathrm{mEq} / \mathrm{L}$, bicarbonate $17 \mathrm{mEq} / \mathrm{L}$, chloride $107 \mathrm{mmol} / \mathrm{L}$, potassium $3.5 \mathrm{mmol} / \mathrm{L}$, ionized calcium $1.09 \mathrm{mmol} / \mathrm{L}$ (normal: 1.18-1.29 mmol/L), magnesium $1.7 \mathrm{mg} / \mathrm{dl}$ (normal: 1.8-2.4 $\mathrm{mg} / \mathrm{dl}$ ).X-rays of the hand demonstrated no bony abnormality or foreign body. A 12-lead EKG was performed which demonstrated a normal sinus rhythm, without peaked t-waves, and normal intervals including the corrected Q-T interval.

Based on the suspicion for HFA exposure, in conjunction with a medical toxicologist, we administered topical calcium gluconate as initial therapy. Additionally were pleted calcium and magnesium levels and began hourly electrolyte testing and continuous telemetry.

A $2.5 \%$ topical calcium gluconate solution was prepared by mixing 1 gram of calcium gluconate and $40 \mathrm{ml}$ of $\mathrm{K}-\mathrm{Y}^{\circledast}$ Jelly.applied to both hands with standard latex gloves placed on the patient to keep solution in place. The patient also received 1 gram of magnesium gluconate, 400 $\mathrm{mg}$ of magnesium oxide orally, and two 1-gram boluses of calcium gluconate intravenously 4 hours apart.

Despite the intravenous morphine bolus, the patient didn't begin to report pain control until 30 minutes after application of the

*Corresponding author: Robert Bassett, FAAEM, Medical Toxicology Fellow, Department of Emergency Medicine, Einstein Medical Center, USA, Tel: 215-4566501; Fax: 215-456-7834; E-mail: bassettr@einstein.edu

Received August 30, 2014; Accepted April 11, 2015; Published April 13, 2015

Citation: Bassett RA(2015) Finger Pain out of Proportion to Exam: A Case of Occult Hydrofluoric Acid Toxicity. Med chem 5: 152-153. doi:10.4172/2161-0444.1000257

Copyright: @ 2015 Bassett RA. This is an open-access article distributed under the terms of the Creative Commons Attribution License, which permits unrestricted use, distribution, and reproduction in any medium, provided the original author and source are credited. 
topical calcium gluconate solution. The patient was admitted for serial electrolyte testing and cardiac monitoring. The patient was discharged 24 hours later with normal electrolytes and excellent pain control.

\section{Discussion}

Hydrofluoric acid was first reportedly prepared in the $17^{\text {th }}$ century becoming commercially available in 1931 for glass etching [1]. Although considered a weak acid because of its lower dissociation constant it's highly corrosive dissolving powers are well recognized [2]. HFA is used in many areas of industry including oil refining, glass etching, and industrial cleaning particularly rust removal.This last use is one of the reasons it is so readily available to the general public.

Owing to a high permeability coefficient, HFA is able to penetrate deep into human tissue before dissociation into hydrogen and fluoride ions [3]. These electronegative fluoride ions have a high affinity for calcium and magnesium resulting in rapid depletion and potentially severe cardiac sequelae [4]. The electrolyte depletion is dose dependent with the most severe cases resulting from high concentration HFA exposure.QT interval prolongation is a potential early manifestation of hypocalcemia and hypomagnesemia. The uncorrected depletion of these vital electrolytes can result in fatal ventricular dysrhythmias including ventricular tachycardia and ventricular fibrillation. Unfortunately, even correctly identifying this toxicity does not always prevent fatal outcomes. There are many documented cases of ventricular dysrhythmias refractory to treatment with intravenous calcium and magnesium [5-7].

Epidemiologically, hydrofluoric acid poisoning is not very common.Only 591 cases of single exposures were reported in the 2011 annual report by the American Association of Poison Control Centers. However,HFA exposures have the tendency to be significant showing that nearly 25\% (142/591) were reported as "Moderate" or "Major" [8].

The cases most likely to have major outcomes or severe toxicity are the larger volume and high concentration exposures.Fortunately, the highest concentrations of HFA are typically reserved for industrial products used by technicians who are more likely to be aware of the dangers of this chemical product.However, the householduse of industrial chemicals is a particularly dangerous situation. Highconcentration HFA exposures typically have rapid onset of severe symptoms.Thus, the greater the exposure, the sooner the symptoms. These cases rarely present a diagnostic dilemma.

Unfortunately, the lower concentration HFA products, those most readily available to the general public, will often present with few clues to the diagnosis.In the case presented, our patient had few historical indices. The term "cleaner" especially without the description of rust remover, is not likely to prompt an association with HFA.The lack of physical exam findings could have easily been dismissed as an indication of a non-serious injury.The localization of isolated fingertip pain could also contribute to a cognitive error (e.g. triage cueing, visceral bias) of underestimating the potential systemic toxicity [9].

This case perfectly illustrates the importance of considering the potential of HFA toxicity in dermal exposures to unknown "cleaning" agents.The hallmark dyad of 1) delayed onset pain and 2) pain that is out of proportion to the physical exam findings should trigger consideration of a possible HFA exposure. The surrogate markers of low serum calcium and magnesium will help to confirm the diagnosis in a timely manner.Although serum fluoride levels can be measured, they are often unavailable in a time frame that will be clinically meaningful to a patient or provider.Additionally, a screening electrocardiogram should be reviewed for early signs of cardiotoxicity such as QT interval
prolongation.Telemetry should be performed until it is clear that the patient's potential for ventricular dysrhythmia has subsided.Once calcium and magnesium levels have stabilized within normal limits further telemetry is unnecessary.

Treatment of HFA poisoning can be both therapeutic and diagnostic.The introduction of calcium at the exposure site will often serve to confirm the diagnosis as heralded by a rapid reduction in pain. The chloride based calcium combinations are considered too noxious to local tissue and thus should be avoided. Calcium gluconate topical solutions can dramatically reverse a patient's pain level.By restoring the depleted calcium levels, the neuroexcitation and vasospasm that are believed to be the etiology of the pain response, can be reversed [10]. Fortunately, the efficacy of the antidote can be measured clinically. The mitigation of pain is predicative of calcium repletion. Therefore, most textbooks recommend that low-concentration HFA exposure patient be observed for 4-6 hours after they achieve pain control. If they remain pain free no further testing is necessarily required and they may be discharged for outpatient follow up burn care $[11,12]$.

\section{Conclusion}

Low concentration HFA exposures can present to the emergency department with subtle signs and symptoms creating a diagnostic dilemma.The delayed onset of physical exam findings should prompt consideration of HFA toxicity in patients who present with finger pain that report recent exposure to any "cleaning" agent.A trial of calcium gluconate in unknown finger pain may be both therapeutic and diagnostic as complete resolution of symptoms is highly suggestive of occult HFA exposure.

\section{References}

1. Caravati EM (1988) Acute hydrofluoric acid exposure.Am J Emerg Med 6: 143150.

2. Ayotte $P$, Hebert M, Marchand $P$ (2005) Why is hydrofluoric acid a weak acid? J Chem Phys 123: 184501

3. Gutknecht J, Walter A (1981) Hydrofluoric and nitric acid transport through lipid bilayer membranes. Biochim Biophys Acta 644: 153-156.

4. McClure FJ (1933) A review of fluorine and its physiologic effects. Physiol Rev 13: $277-300$

5. Mullett T, Zoeller T, Bingham H, Pepine CJ, Prida XE, et al. (1987) Fatal hydrofluoric acid cutaneous exposure with refractory ventricular fibrillation. $J$ Burn Care Rehabil 8: 216-219.

6. Mayer TG, Gross PL (1985) Fatal systemic fluorosis due to hydrofluoric acid burns. Ann Emerg Med 14: 149-153.

7. Mullett T, Zoeller T, Bingham H, Pepine CJ, Prida XE, et al. (1987) Fatal hydrofluoric acid cutaneous exposure with refractory ventricular fibrillation.J Burn Care Rehabil 8: 216-219.

8. Bronstein AC,Spyker DA, Cantilena LR Jr, Rumack BH, Dart RC (2012) 2011 Annual report of the American Association of Poison Control Centers' National Poison Data System (NPDS): 29th Annual Report. Clin Toxicol (Phila) 50: 9111164.

9. Bronstein AC, Spyker DA, Cantilena LR, Rumack BH, Dart RC (2012) Clinical Toxicology: The Official Journal of the American Academy of Clinical Toxicology \& European Association of Poisons Centres\& Clinical Toxicologists 50: $911-1164$.

10. Croskerry $P$ (2003) The importance of cognitive errors in diagnosis and strategies to minimize them. Acad Med 78: 775-780.

11. Su M (2011) Chapter 105. Hydrofluoric Acid and Fluorides. In: Su M, ed. Goldfrank's Toxicologic Emergencies. 9th ed. New York.

12. Hydrofluoric Acid and Ammonium Bifluoride (2000) In: Dart, Richard C. eds 5-Minute Toxicology Consult. 1st Edition. 227 East Washington Square Philadelphia, PA 19106-3780 USA, LWW.com:Lippincott Williams \& Wilkins. 